Article

\title{
On a Generalized Langevin Type Nonlocal Fractional Integral Multivalued Problem
}

\author{
Ahmed Alsaedi ${ }^{1}\left(\mathbb{D}\right.$, Bashir Ahmad ${ }^{1, *} \mathbb{C}$, Madeaha Alghanmi ${ }^{1}$ and Sotiris K. Ntouyas ${ }^{1,2}$ (I) \\ 1 Nonlinear Analysis and Applied Mathematics (NAAM)-Research Group, Department of Mathematics, \\ Faculty of Science, King Abdulaziz University, P.O. Box 80203, Jeddah 21589, Saudi Arabia; \\ aalsaedi@hotmail.com (A.A.); madeaha@hotmail.com (M.A.); sntouyas@uoi.gr (S.K.N.) \\ 2 Department of Mathematics, University of Ioannina, 45110 Ioannina, Greece \\ * Correspondence: bmuhammed@kau.edu.sa
}

Received: 29 August 2019; Accepted: 21 October 2019; Published: 25 October 2019

\begin{abstract}
We establish sufficient criteria for the existence of solutions for a nonlinear generalized Langevin-type nonlocal fractional-order integral multivalued problem. The convex and non-convex cases for the multivalued map involved in the given problem are considered. Our results rely on Leray-Schauder nonlinear alternative for multivalued maps and Covitz and Nadler's fixed point theorem. Illustrative examples for the main results are included.
\end{abstract}

Keywords: differential inclusions; Caputo-type fractional derivative; fractional integral; existence; fixed point

\section{Introduction}

Fractional calculus is the extension of classical calculus which deals with differential and integral operators of fractional order. It has evolved into a significant and popular branch of mathematical analysis owing to its extensive applications in the mathematical modeling of applied and technical problems. The literature on fractional calculus is now much enriched and covers a wide range of interesting results, for instance [1-6]. For a comprehensive treatment of Hadamard-type fractional differential equations and inclusions, we refer the reader to the text [7].

The Langevin equation is found to be an effective tool to describe stochastic problems in fluctuating situations. A modified type of this equation is used in various functional approaches for fractal media. A variety of boundary value problems involving the Langevin equation have been investigated by several authors. In [8], existence and uniqueness results for a nonlinear Langevin equation involving two fractional orders supplemented with three-point boundary conditions were obtained. An impulsive boundary value problem for a nonlinear Langevin equation involving two different fractional derivatives was investigated in [9]. Some existing results for Langevin fractional differential inclusions with two indices were derived in [10]. In [11], the authors proved the existence of and uniqueness results for an anti-periodic boundary value problem of a system of Langevin fractional differential equations. In [12], the authors investigated a nonlinear fractional Langevin equation with anti-periodic boundary conditions by applying coupled fixed point theorems. In a recent work [13], the authors obtained some existence results for a fractional Langevin equation with nonlinearity depending on Riemann-Liouville fractional integral, and complemented with nonlocal multi-point and multi-strip boundary conditions.

In the present paper, we study the existence of solutions for a nonlinear generalized Langevin type nonlocal fractional-order integral multivalued problem given by 


$$
\left\{\begin{array}{l}
{ }_{c}^{\rho} D_{a+}^{\alpha}\left({ }_{c}^{\rho} D_{a+}^{\beta}+\lambda\right) x(t) \in F(t, x(t)), \quad t \in J:=[a, T], \quad \lambda \in \mathbb{R}, \\
x(a)=0, \quad x(\eta)=0, \quad x(T)=\mu^{\rho} I_{a+}^{\gamma} x(\xi), \quad a<\eta<\xi<T, \mu \in \mathbb{R},
\end{array}\right.
$$

where ${ }_{c}^{\rho} D_{a+,}^{\alpha}{ }_{c}^{\rho} D_{a+}^{\beta}$ denote the Caputo-type generalized fractional differential operators of order $1<\alpha \leq 2,0<\beta<1, \rho>0$, respectively, $F:[a, T] \times \mathbb{R} \rightarrow \mathcal{P}(\mathbb{R})$ is a multi-valued map $(\mathcal{P}(\mathbb{R})$ is the family of all nonempty subsets of $\mathbb{R}),{ }^{\rho} I_{a+}^{\gamma}$ is the generalized fractional integral operator of order $\gamma>0$ and $\rho>0$. Here we emphasize that the single-valued analogue of the problem (1) was discussed in [14].

The rest of the paper is arranged as follows. The background material related to our work is outlined in Section 3. The existence results for the problem (1) are presented in Section 3. The first result for the problem (1), associated with the convex valued mutivalued map, is derived with the aid of Leray-Schauder nonlinear alternative for multivalued maps, while the result for non-convex valued map for the problem (1) is proved by applying a fixed point theorem due to Covitz and Nadler. Section 4 contains the illustrative examples for the main results. We summarize the work established in this paper, and its implications, in the last section.

\section{Preliminaries}

Define by $X_{c}^{p}(a, b)$ the space of all complex-valued Lebesgue measurable functions $\phi$ on $(a, b)$ equipped with the norm:

$$
\|\phi\|_{X_{c}^{p}}=\left(\int_{a}^{b}\left|x^{c} \phi(x)\right|^{p} \frac{d x}{x}\right)^{1 / p}<\infty, c \in \mathbb{R}, 1 \leq p \leq \infty .
$$

Let $A C_{\delta}^{n}[a, b]$ denote the class of all absolutely continuous functions $g$ possessing $\delta^{n-1}$-derivative $\left(\delta^{n-1} g \in A C([a, b], \mathbb{R})\right)$, endowed with the norm $\|g\|_{A C_{\delta}^{n}}=\sum_{k=0}^{n-1}\left\|\delta^{k} g\right\|_{C}$.

Definition 1. The left-sided and right-sided generalized fractional integrals for $g \in X_{c}^{p}(a, b)$ of order $\beta>0$ and $\rho>0$, denoted by ${ }^{\rho} I_{a+}^{\beta} g$ and ${ }^{\rho} I_{b-}^{\beta} g$ respectively, are defined by [15]

$$
\begin{aligned}
& \left({ }^{\rho} I_{a+}^{\beta} g\right)(t)=\frac{\rho^{1-\beta}}{\Gamma(\beta)} \int_{a}^{t} \frac{s^{\rho-1}}{\left(t^{\rho}-s^{\rho}\right)^{1-\beta}} g(s) d s,-\infty<a<t<b<\infty, \\
& \left({ }^{\rho} I_{b-}^{\beta} g\right)(t)=\frac{\rho^{1-\alpha}}{\Gamma(\beta)} \int_{t}^{b} \frac{s^{\rho-1}}{\left(s^{\rho}-t^{\rho}\right)^{1-\beta}} g(s) d s,-\infty<a<t<b<\infty .
\end{aligned}
$$

Definition 2. Let $\beta>0, n=[\beta]+1$ and $\rho>0$. We define the generalized fractional derivatives, associated with the generalized fractional integrals (2) and (3), for $0 \leq a<t<b<\infty$, as follows [16]:

$$
\begin{gathered}
\left({ }^{\rho} D_{a+}^{\beta} g\right)(t)=\left(t^{1-\rho} \frac{d}{d t}\right)^{n}\left({ }^{\rho} I_{a+}^{n-\beta} g\right)(t)=\frac{\rho^{\beta-n+1}}{\Gamma(n-\beta)}\left(t^{1-\rho} \frac{d}{d t}\right)^{n} \int_{a}^{t} \frac{s^{\rho-1}}{\left(t^{\rho}-s^{\rho}\right)^{\beta-n+1}} g(s) d s, \\
\left({ }^{\rho} D_{b-}^{\beta} g\right)(t)=\left(-t^{1-\rho} \frac{d}{d t}\right)^{n}\left({ }^{\rho} I_{b-}^{n-\beta} g\right)(t)=\frac{\rho^{\beta-n+1}}{\Gamma(n-\beta)}\left(-t^{1-\rho} \frac{d}{d t}\right)^{n} \int_{t}^{b} \frac{s^{\rho-1}}{\left(s^{\rho}-t^{\rho}\right)^{\beta-n+1}} g(s) d s,
\end{gathered}
$$

provided the integrals in the above expressions exist. 
Definition 3. Let $g \in A C_{\delta}^{n}[a, b]$ and $\beta>0, n=[\beta]+1$. Then the Caputo-type generalized fractional derivatives ${ }_{c}^{\rho} D_{a+g}^{\beta} g$ and ${ }_{c}^{\rho} D_{b-}^{\beta} g$ are respectively defined via (4) and (5) by [17]

$$
\begin{gathered}
{ }_{c}^{\rho} D_{a+}^{\beta} g(x)={ }^{\rho} D_{a+}^{\beta}\left[g(t)-\sum_{k=0}^{n-1} \frac{\delta^{k} g(a)}{k !}\left(\frac{t^{\rho}-a^{\rho}}{\rho}\right)^{k}\right](x), \delta=x^{1-\rho} \frac{d}{d x}, \\
{ }_{c}^{\rho} D_{b-\delta}^{\beta} g(x)={ }^{\rho} D_{b-}^{\beta}\left[g(t)-\sum_{k=0}^{n-1} \frac{(-1)^{k} \delta^{k} g(b)}{k !}\left(\frac{b^{\rho}-t^{\rho}}{\rho}\right)^{k}\right](x), \delta=x^{1-\rho} \frac{d}{d x} .
\end{gathered}
$$

Remark 1. The left and right generalized Caputo derivatives of order $\beta$ for $g \in A C_{\delta}^{n}[a, b]$, are respectively given by [17]

$$
\begin{gathered}
\rho_{c} D_{a+g}^{\beta} g(t)=\frac{1}{\Gamma(n-\beta)} \int_{a}^{t}\left(\frac{t^{\rho}-s^{\rho}}{\rho}\right)^{n-\beta-1} \frac{\left(\delta^{n} g\right)(s) d s}{s^{1-\rho}}, \\
{ }_{c}^{\rho} D_{b-}^{\beta} g(t)=\frac{1}{\Gamma(n-\beta)} \int_{t}^{b}\left(\frac{s^{\rho}-t^{\rho}}{\rho}\right)^{n-\alpha-1} \frac{(-1)^{n}\left(\delta^{n} g\right)(s) d s}{s^{1-\rho}} .
\end{gathered}
$$

Lemma 1. Let $g \in A C_{\delta}^{n}[a, b]$ or $C_{\delta}^{n}[a, b]$. Then, for $\beta \in \mathbb{R}$, the following results hold [17]:

$$
\begin{gathered}
\rho I_{a+c}^{\beta \rho} D_{a+\delta}^{\beta} g(x)=g(x)-\sum_{k=0}^{n-1} \frac{\left(\delta^{k} g\right)(a)}{k !}\left(\frac{x^{\rho}-a^{\rho}}{\rho}\right)^{k}, \\
{ }^{\rho} I_{b-c}^{\beta} \rho_{b}^{\rho} D_{b-}^{\beta} g(x)=g(x)-\sum_{k=0}^{n-1} \frac{(-1)^{k}\left(\delta^{k} g\right)(a)}{k !}\left(\frac{b^{\rho}-x^{\rho}}{\rho}\right)^{k} .
\end{gathered}
$$

In particular, for $0<\beta \leq 1$, we have

$$
{ }^{\rho} I_{a+c}^{\beta \rho} D_{a+}^{\beta} g(x)=g(x)-g(a),{ }^{\rho} I_{b^{-}}^{\beta}{ }^{\rho} D_{b^{-}}^{\beta} g(x)=g(x)-g(b) .
$$

We need the following known lemma [14] in the sequel.

Lemma 2. Let $h \in C([a, T], \mathbb{R})$ and $x \in A C_{\delta}^{3}(J)$. Then the unique solution of linear problem:

$$
\left\{\begin{array}{l}
{ }_{c}^{\rho} D_{a+}^{\alpha}\left({ }_{c}^{\rho} D_{a+}^{\beta}+\lambda\right) x(t)=h(t), \quad t \in J:=[a, T], \\
x(a)=0, x(\eta)=0, x(T)=\mu^{\rho} I_{a+}^{\gamma} x(\xi), \quad a<\eta<\xi<T,
\end{array}\right.
$$

is given by:

$$
\begin{aligned}
x(t)= & { } I_{a+}^{\alpha+\beta} h(t)-\lambda^{\rho} I_{a+}^{\beta} x(t)+\frac{\left(t^{\rho}-a^{\rho}\right)^{\beta}\left(\eta^{\rho}-t^{\rho}\right)}{\rho^{\beta+1} \Gamma(\beta+2) \Omega}\left\{{ }^{\rho} I_{a+}^{\alpha+\beta} h(T)-\lambda^{\rho} I_{a+}^{\beta} x(T)\right. \\
& \left.-\mu^{\rho} I_{a+}^{\alpha+\beta+\gamma} h(\xi)+\mu \lambda^{\rho} I_{a+}^{\beta+\gamma} x(\xi)\right\}-\frac{\left(t^{\rho}-a^{\rho}\right)^{\beta}}{\Omega\left(\eta^{\rho}-a^{\rho}\right)^{\beta}}\left(\frac{\left(T^{\rho}-a^{\rho}\right)^{\beta}\left(T^{\rho}-t^{\rho}\right)}{\rho^{\beta+1} \Gamma(\beta+2)}\right. \\
& \left.-\frac{\mu\left(\xi^{\rho}-a^{\rho}\right)^{\beta+\gamma}\left[(\beta+1)\left(\xi^{\rho}-t^{\rho}\right)-\gamma\left(t^{\rho}-a^{\rho}\right)\right]}{\rho^{\beta+\gamma+1} \Gamma(\beta+\gamma+2)(\beta+1)}\right)\left\{{ }^{\rho} I_{a+}^{\alpha+\beta} h(\eta)-\lambda^{\rho} I_{a+}^{\beta} x(\eta)\right\},
\end{aligned}
$$

where it is assumed that

$$
\Omega=\left[\frac{\left(T^{\rho}-a^{\rho}\right)^{\beta}\left(T^{\rho}-\eta^{\rho}\right)}{\rho^{\beta+1} \Gamma(\beta+2)}-\frac{\mu\left(\xi^{\rho}-a^{\rho}\right)^{\beta+\gamma}\left[(\beta+1)\left(\xi^{\rho}-\eta^{\rho}\right)-\gamma\left(\eta^{\rho}-a^{\rho}\right)\right]}{\rho^{\beta+\gamma+1} \Gamma(\beta+\gamma+2)(\beta+1)}\right] \neq 0 .
$$

\section{Main Results}

We begin this section with the definition of a solution for the multi-valued problem (1). 
Definition 4. A function $x \in C(J, \mathbb{R})$ is called a solution of the problem (1) if we can find a function $v \in L^{1}(J, \mathbb{R})$ with $v(t) \in F(t, x)$ a.e. on J such that $x(a)=0, x(\eta)=0, x(T)=\mu^{\rho} I_{a+}^{\gamma} x(\xi)$ and

$$
\begin{aligned}
x(t)= & \rho I_{a+}^{\alpha+\beta} v(t)-\lambda^{\rho} I_{a+}^{\beta} x(t)+\frac{\left(t^{\rho}-a^{\rho}\right)^{\beta}\left(\eta^{\rho}-t^{\rho}\right)}{\rho^{\beta+1} \Gamma(\beta+2) \Omega}\left\{{ }^{\rho} I_{a+}^{\alpha+\beta} v(T)-\lambda^{\rho} I_{a+}^{\beta} x(T)\right. \\
& \left.-\mu^{\rho} I_{a+}^{\alpha+\beta+\gamma} v(\xi)+\mu \lambda^{\rho} I_{a+}^{\beta+\gamma} x(\xi)\right\}-\frac{\left(t^{\rho}-a^{\rho}\right)^{\beta}}{\Omega\left(\eta^{\rho}-a^{\rho}\right)^{\beta}}\left(\frac{\left(T^{\rho}-a^{\rho}\right)^{\beta}\left(T^{\rho}-t^{\rho}\right)}{\rho^{\beta+1} \Gamma(\beta+2)}\right. \\
& \left.-\frac{\mu\left(\xi^{\rho}-a^{\rho}\right)^{\beta+\gamma}\left[(\beta+1)\left(\xi^{\rho}-t^{\rho}\right)-\gamma\left(t^{\rho}-a^{\rho}\right)\right]}{\rho^{\beta+\gamma+1} \Gamma(\beta+\gamma+2)(\beta+1)}\right)\left\{{ }^{\rho} I_{a+}^{\alpha+\beta} v(\eta)-\lambda^{\rho} I_{a+}^{\beta} x(\eta)\right\} .
\end{aligned}
$$

For the sake of computational convenience, we set

$$
\begin{aligned}
\Lambda_{1}= & \frac{\left(T^{\rho}-a^{\rho}\right)^{\alpha+\beta}}{\rho^{\alpha+\beta} \Gamma(\alpha+\beta+1)}\left[1+\frac{\zeta_{1}}{\rho^{\beta+1} \Gamma(\beta+2)|\Omega|}\right]+\frac{|\mu|\left(\xi^{\rho}-a^{\rho}\right)^{\alpha+\beta+\gamma} \zeta_{1}}{\rho^{\alpha+2 \beta+\gamma+1} \Gamma(\alpha+\beta+\gamma+1) \Gamma(\beta+2)|\Omega|} \\
& +\frac{\left(\eta^{\rho}-a^{\rho}\right)^{\alpha} \zeta_{2}}{\rho^{\alpha+\beta} \Gamma(\alpha+\beta+1)|\Omega|}, \\
\Lambda_{2}= & \frac{|\lambda|\left(T^{\rho}-a^{\rho}\right)^{\beta}}{\rho^{\beta} \Gamma(\beta+1)}\left[1+\frac{\zeta_{1}}{\rho^{\beta+1} \Gamma(\beta+2)|\Omega|}\right]+\frac{|\mu||\lambda|\left(\xi^{\rho}-a^{\rho}\right)^{\beta+\gamma} \zeta_{1}}{\rho^{2 \beta+\gamma+1} \Gamma(\beta+\gamma+1) \Gamma(\beta+2)|\Omega|} \\
& \quad+\frac{|\lambda| \zeta_{2}}{\rho^{\beta} \Gamma(\beta+1)|\Omega|},
\end{aligned}
$$

where

$$
\begin{gathered}
\zeta_{1}:=\max _{t \in[a, T]}\left|\left(t^{\rho}-a^{\rho}\right)^{\beta}\left(\eta^{\rho}-t^{\rho}\right)\right|, \\
\zeta_{2}:=\max _{t \in[a, T]}\left|\left(t^{\rho}-a^{\rho}\right)^{\beta}\left[\frac{\left(T^{\rho}-a^{\rho}\right)^{\beta}\left(T^{\rho}-t^{\rho}\right)}{\rho^{\beta+1} \Gamma(\beta+2)}-\frac{\mu\left(\xi^{\rho}-a^{\rho}\right)^{\beta+\gamma}\left[(\beta+1)\left(\xi^{\rho}-t^{\rho}\right)-\gamma\left(t^{\rho}-a^{\rho}\right)\right]}{\rho^{\beta+\gamma+1} \Gamma(\beta+\gamma+2)(\beta+1)}\right]\right| .
\end{gathered}
$$

We define the set of selections of $F$ by $S_{F, x}:=\left\{y \in L^{1}(J, \mathbb{R}): y(t) \in F(t, x(t))\right.$ on $\left.J\right\}$ for each $x \in C(J, \mathbb{R})$.

\subsection{The Upper Semicontinuous Case}

In the following result, we assume that the multivalued map $F$ is convex-valued and apply Leray-Schauder nonlinear alternative for multivalued maps [18] to prove the existence of solutions for the problem at hand.

Theorem 1. Assume that:

$\left(A_{1}\right) F: J \times \mathbb{R} \rightarrow \mathcal{P}_{c p, c}(\mathbb{R})$ is $L^{1}$-Carathéodory, where $\mathcal{P}_{c p, c}(\mathbb{R})=\{\mathcal{Y} \in \mathcal{P}(\mathbb{R}): \mathcal{Y}$ is compact and convex $\} ;$

$\left(A_{2}\right)$ there exist a function $P \in C\left(J, \mathbb{R}^{+}\right)$and a continuous nondecreasing function $Q:[0, \infty) \rightarrow(0, \infty)$ such that $\|F(t, x)\|_{\mathcal{P}}:=\sup \{|y|: y \in F(t, x)\} \leq P(t) Q(|x|)$ for each $(t, x) \in J \times \mathbb{R}$;

$\left(A_{3}\right)$ there exists a constant $M>0$ such that

$$
\frac{\left(1-\Lambda_{2}\right) M}{\Lambda_{1}\|P\| Q(M)}>1, \quad \Lambda_{2}<1,
$$

where $\Lambda_{1}$ and $\Lambda_{2}$ are respectively given by (14) and (15).

Then the problem (1) has at least one solution on J. 
Proof. Let us first convert the problem (1) into a fixed point problem by introducing a multivalued map: $N: C(J, \mathbb{R}) \rightarrow \mathcal{P}(C(J, \mathbb{R}))$ as

$$
N(x)=\left\{\begin{array}{l}
h \in C(J, \mathbb{R}): \\
h(t)=\left\{\begin{array}{l}
{ }^{\rho} I_{a+}^{\alpha+\beta} v(t)-\lambda^{\rho} I_{a+}^{\beta} x(t) \\
+\frac{\left(t^{\rho}-a^{\rho}\right)^{\beta}\left(\eta^{\rho}-t^{\rho}\right)}{\rho^{\beta+1} \Gamma(\beta+2) \Omega}\left\{{ }^{\rho} I_{a+}^{\alpha+\beta} v(T)-\lambda^{\rho} I_{a+}^{\beta} x(T)\right. \\
\left.-\mu^{\rho} I_{a+}^{\alpha+\beta+\gamma} v(\xi)+\mu \lambda^{\rho} I_{a+}^{\beta+\gamma} x(\xi)\right\}-\frac{\left(t^{\rho}-a^{\rho}\right)^{\beta}}{\Omega\left(\eta^{\rho}-a^{\rho} \beta\right.}\left(\frac{\left(T^{\rho}-a^{\rho}\right)^{\beta}\left(T^{\rho}-t^{\rho}\right)}{\rho^{\beta+1} \Gamma(\beta+2)}\right. \\
\left.-\frac{\mu\left(\xi^{\rho}-a^{\rho}\right)^{\beta+\gamma}\left[(\beta+1)\left(\xi^{\rho}-t^{\rho}\right)-\gamma\left(t^{\rho}-a^{\rho}\right)\right]}{\rho^{\beta+\gamma+1} \Gamma(\beta+\gamma+2)(\beta+1)}\right)\{\rho
\end{array} I_{a+}^{\alpha+\beta} v(\eta)-\lambda^{\rho} I_{a+}^{\beta} x(\eta)\right\},
\end{array}\right\}
$$

for $v \in S_{F, x}$.

It is clear that fixed points of $N$ are solutions of problem (1). So we need to verify that the operator $N$ satisfies all the conditions of Leray-Schauder nonlinear alternative [18]. This will be done in several steps.

Step 1. $N(x)$ is convex for each $x \in C(J, \mathbb{R})$.

Indeed, if $h_{1}, h_{2}$ belongs to $N(x)$, then there exist $v_{1}, v_{2} \in S_{F, x}$ such that, for each $t \in J$, we have

$$
\begin{aligned}
h_{i}(t)= & \rho I_{a+}^{\alpha+\beta} v_{i}(t)-\lambda^{\rho} I_{a+}^{\beta} x(t)+\frac{\left(t^{\rho}-a^{\rho}\right)^{\beta}\left(\eta^{\rho}-t^{\rho}\right)}{\rho^{\beta+1} \Gamma(\beta+2) \Omega}\left\{{ }^{\rho} I_{a+}^{\alpha+\beta} v_{i}(T)-\lambda^{\rho} I_{a+}^{\beta} x(T)\right. \\
& \left.-\mu^{\rho} I_{a+}^{\alpha+\beta+\gamma} v_{i}(\xi)+\mu \lambda^{\rho} I_{a+}^{\beta+\gamma} x(\xi)\right\}-\frac{\left(t^{\rho}-a^{\rho}\right)^{\beta}}{\Omega\left(\eta^{\rho}-a^{\rho}\right)^{\beta}}\left(\frac{\left(T^{\rho}-a^{\rho}\right)^{\beta}\left(T^{\rho}-t^{\rho}\right)}{\rho^{\beta+1} \Gamma(\beta+2)}\right. \\
& \left.-\frac{\mu\left(\xi^{\rho}-a^{\rho}\right)^{\beta+\gamma}\left[(\beta+1)\left(\xi^{\rho}-t^{\rho}\right)-\gamma\left(t^{\rho}-a^{\rho}\right)\right]}{\rho^{\beta+\gamma+1} \Gamma(\beta+\gamma+2)(\beta+1)}\right)\{\rho \\
& \left.I_{a+}^{\alpha+\beta} v_{i}(\eta)-\lambda^{\rho} I_{a+}^{\beta} x(\eta)\right\}, i=1,2 .
\end{aligned}
$$

Let $t \in J$ and $\theta \in(0,1)$. Then

$$
\begin{aligned}
& {\left[\theta h_{1}+(1-\theta) h_{2}\right](t)} \\
& =\rho^{\rho} I_{a+}^{\alpha+\beta}\left[\theta v_{1}(s)+(1-\theta) v_{2}(s)\right](t)-\lambda^{\rho} I_{a+}^{\beta} x(t) \\
& +\frac{\left(t^{\rho}-a^{\rho}\right)^{\beta}\left(\eta^{\rho}-t^{\rho}\right)}{\rho^{\beta+1} \Gamma(\beta+2) \Omega}\left\{{ }^{\rho} I_{a+}^{\alpha+\beta}\left[\theta v_{1}(s)+(1-\theta) v_{2}(s)\right](T)-\lambda^{\rho} I_{a+}^{\beta} x(T)\right. \\
& \left.-\mu^{\rho} I_{a+}^{\alpha+\beta+\gamma}\left[\theta v_{1}(s)+(1-\theta) v_{2}(s)\right](\xi)+\mu \lambda^{\rho} I_{a+}^{\beta+\gamma} x(\xi)\right\}-\frac{\left(t^{\rho}-a^{\rho}\right)^{\beta}}{\Omega\left(\eta^{\rho}-a^{\rho}\right)^{\beta}}\left(\frac{\left(T^{\rho}-a^{\rho}\right)^{\beta}\left(T^{\rho}-t^{\rho}\right)}{\rho^{\beta+1} \Gamma(\beta+2)}\right. \\
& \left.-\frac{\mu\left(\xi^{\rho}-a^{\rho}\right)^{\beta+\gamma}\left[(\beta+1)\left(\xi^{\rho}-t^{\rho}\right)-\gamma\left(t^{\rho}-a^{\rho}\right)\right]}{\rho^{\beta+\gamma+1} \Gamma(\beta+\gamma+2)(\beta+1)}\right)\left\{\rho I_{a+}^{\alpha+\beta}\left[\theta v_{1}(s)+(1-\theta) v_{2}(s)\right](\eta)-\lambda^{\rho} I_{a+}^{\beta} x(\eta)\right\} .
\end{aligned}
$$

Since $F$ has convex values ( $S_{F, x}$ is convex), therefore, $\theta h_{1}+(1-\theta) h_{2} \in N(x)$.

Step 2. $N(x)$ maps bounded sets (balls) into bounded sets in $C(J, \mathbb{R})$.

Let $B_{r}=\{x \in C(J, \mathbb{R}):\|x\| \leq r\}$ be a bounded ball in $C(J, \mathbb{R})$, where $r$ is a positive number. Then, for each $h \in N(x), x \in B_{r}$, there exists $v \in S_{F, x}$ such that

$$
\begin{aligned}
h(t)= & { }^{\rho} I_{a+}^{\alpha+\beta} v(t)-\lambda^{\rho} I_{a+}^{\beta} x(t)+\frac{\left(t^{\rho}-a^{\rho}\right)^{\beta}\left(\eta^{\rho}-t^{\rho}\right)}{\rho^{\beta+1} \Gamma(\beta+2) \Omega}\left\{{ }^{\rho} I_{a+}^{\alpha+\beta} v(T)-\lambda^{\rho} I_{a+}^{\beta} x(T)\right. \\
& \left.-\mu^{\rho} I_{a+}^{\alpha+\beta+\gamma} v(\xi)+\mu \lambda^{\rho} I_{a+}^{\beta+\gamma} x(\xi)\right\}-\frac{\left(t^{\rho}-a^{\rho}\right)^{\beta}}{\Omega\left(\eta^{\rho}-a^{\rho}\right)^{\beta}}\left(\frac{\left(T^{\rho}-a^{\rho}\right)^{\beta}\left(T^{\rho}-t^{\rho}\right)}{\rho^{\beta+1} \Gamma(\beta+2)}\right. \\
& \left.-\frac{\mu\left(\xi^{\rho}-a^{\rho}\right)^{\beta+\gamma}\left[(\beta+1)\left(\xi^{\rho}-t^{\rho}\right)-\gamma\left(t^{\rho}-a^{\rho}\right)\right]}{\rho^{\beta+\gamma+1} \Gamma(\beta+\gamma+2)(\beta+1)}\right)\left\{{ }^{\rho} I_{a+}^{\alpha+\beta} v(\eta)-\lambda^{\rho} I_{a+}^{\beta} x(\eta)\right\} .
\end{aligned}
$$


In view of $\left(H_{2}\right)$, for each $t \in J$, we find that

$$
\begin{aligned}
|h(t)| \leq & { }^{\rho} I_{a+}^{\alpha+\beta}|v(t)|+|\lambda|{ }^{\rho} I_{a+}^{\beta}|x(t)|+\frac{\left|\left(t^{\rho}-a^{\rho}\right)^{\beta}\left(\eta^{\rho}-t^{\rho}\right)\right|}{\rho^{\beta+1} \Gamma(\beta+2)|\Omega|}\left\{{ }^{\rho} I_{a+}^{\alpha+\beta}|v(T)|+\lambda^{\rho} I_{a+}^{\beta}|x(T)|\right. \\
& \left.+|\mu|^{\rho} I_{a+}^{\alpha+\beta+\gamma}|v(\xi)|+|\mu \lambda|{ }^{\rho} I_{a+}^{\beta+\gamma}|x(\xi)|\right\}+\mid \frac{\left(t^{\rho}-a^{\rho}\right)^{\beta}}{\Omega\left(\eta^{\rho}-a^{\rho}\right)^{\beta}}\left(\frac{\left(T^{\rho}-a^{\rho}\right)^{\beta}\left(T^{\rho}-t^{\rho}\right)}{\rho^{\beta+1} \Gamma(\beta+2)}\right. \\
& \left.+\frac{\mu\left(\xi^{\rho}-a^{\rho}\right)^{\beta+\gamma}\left[(\beta+1)\left(\xi^{\rho}-t^{\rho}\right)-\gamma\left(t^{\rho}-a^{\rho}\right)\right]}{\rho^{\beta+\gamma+1} \Gamma(\beta+\gamma+2)(\beta+1)}\right) \mid\left\{{ }^{\rho} I_{a+}^{\alpha+\beta}|v(\eta)|+|\lambda|{ }^{\rho} I_{a+}^{\beta}|x(\eta)|\right\} \\
\leq & \|P\| Q(\|x\|)\left(\frac{\left(T^{\rho}-a^{\rho}\right)^{\alpha+\beta}}{\rho^{\alpha+\beta} \Gamma(\alpha+\beta+1)}\left[1+\frac{\zeta_{1}}{\rho^{\beta+1} \Gamma(\beta+2)|\Omega|}\right]\right. \\
& \left.+\frac{|\mu|\left(\xi^{\rho}-a^{\rho}\right)^{\alpha+\beta+\gamma} \zeta_{1}}{\rho^{\alpha+2 \beta+\gamma+1} \Gamma(\alpha+\beta+\gamma+1) \Gamma(\beta+2)|\Omega|}+\frac{\left(\eta^{\rho}-a^{\rho}\right)^{\alpha} \zeta_{2}}{\rho^{\alpha+\beta} \Gamma(\alpha+\beta+1)|\Omega|}\right) \\
& +\|x\|\left(\frac{|\lambda|\left(T^{\rho}-a^{\rho}\right)^{\beta}}{\rho^{\beta} \Gamma(\beta+1)}\left[1+\frac{\zeta}{\rho^{\beta+1} \Gamma(\beta+2)|\Omega|}\right]\right. \\
& \left.+\frac{|\mu \| \lambda|\left(\xi^{\rho}-a^{\rho}\right)^{\beta+\gamma} \zeta_{1}}{\rho^{2 \beta+\gamma+1} \Gamma(\beta+\gamma+1) \Gamma(\beta+2)|\Omega|}+\frac{|\lambda| \zeta_{2}}{\rho^{\beta} \Gamma(\beta+1)|\Omega|}\right) \\
= & \Lambda_{1}\|P\| Q(\|x\|)+\Lambda_{2}\|x\|,
\end{aligned}
$$

which leads to $\|h\| \leq \Lambda_{1}\|P\| Q(r)+\Lambda_{2} r$.

Step 3. $N(x)$ maps bounded sets into equicontinuous sets of $C(J, \mathbb{R})$.

Let $x$ be any element in $B_{r}$ and $h \in N(x)$. Then there exists a function $v \in S_{F, x}$ such that, for each $t \in J$ we have

$$
\begin{aligned}
h(t)= & \rho_{I_{a+}^{\alpha+\beta}}^{\alpha+\beta}(t)-\lambda^{\rho} I_{a+}^{\beta} x(t)+\frac{\left(t^{\rho}-a^{\rho}\right)^{\beta}\left(\eta^{\rho}-t^{\rho}\right)}{\rho^{\beta+1} \Gamma(\beta+2) \Omega}\left\{{ }^{\rho} I_{a+}^{\alpha+\beta}{ }_{v}(T)-\lambda^{\rho} I_{a+}^{\beta} x(T)\right. \\
& \left.-\mu^{\rho} I_{a+}^{\alpha+\beta+\gamma} v(\xi)+\mu \lambda^{\rho} I_{a+}^{\beta+\gamma} x(\xi)\right\}-\frac{\left(t^{\rho}-a^{\rho}\right)^{\beta}}{\Omega\left(\eta^{\rho}-a^{\rho}\right)^{\beta}}\left(\frac{\left(T^{\rho}-a^{\rho}\right)^{\beta}\left(T^{\rho}-t^{\rho}\right)}{\rho^{\beta+1} \Gamma(\beta+2)}\right. \\
& \left.-\frac{\mu\left(\xi^{\rho}-a^{\rho}\right)^{\beta+\gamma}\left[(\beta+1)\left(\xi^{\rho}-t^{\rho}\right)-\gamma\left(t^{\rho}-a^{\rho}\right)\right]}{\rho^{\beta+\gamma+1} \Gamma(\beta+\gamma+2)(\beta+1)}\right)\left\{{ }^{\rho} I_{a+}^{\alpha+\beta} v(\eta)-\lambda^{\rho} I_{a+}^{\beta} x(\eta)\right\} .
\end{aligned}
$$


Let $\tau_{1}, \tau_{2} \in J, \tau_{1}<\tau_{2}$. Then

$$
\begin{aligned}
& \left|h\left(t_{2}\right)-h\left(t_{1}\right)\right| \\
& \leq \mid \frac{\rho^{1-(\alpha+\beta)}}{\Gamma(\alpha+\beta)}\left[\int_{0}^{t_{1}} s^{\rho-1}\left[\left(t_{2}^{\rho}-s^{\rho}\right)^{\alpha+\beta-1}-\left(t_{1}^{\rho}-s^{\rho}\right)^{\alpha+\beta-1}\right] v(s) d s+\int_{t_{1}}^{t_{2}} s^{\rho-1}\left(t_{2}^{\rho}-s^{\rho}\right)^{\alpha+\beta-1} v(s) d s\right] \\
& +\left[\frac{\left(t_{2}^{\rho}-a^{\rho}\right)^{\beta}\left(\eta^{\rho}-t_{2}^{\rho}\right)}{\rho^{\beta+1} \Gamma(\beta+2) \Omega}-\frac{\left(t_{1}^{\rho}-a^{\rho}\right)^{\beta}\left(\eta^{\rho}-t_{1}^{\rho}\right)}{\rho^{\beta+1} \Gamma(\beta+2) \Omega}\right]\left\{{ }^{\rho} I_{a+}^{\alpha+\beta} v(T)-\mu^{\rho} I_{a+}^{\alpha+\beta+\gamma} v(\xi)\right\} \\
& -\left[\frac{\left(t_{2}^{\rho}-a^{\rho}\right)^{\beta}}{\Omega\left(\eta^{\rho}-a^{\rho}\right)^{\beta}}\left[\frac{\left(T^{\rho}-a^{\rho}\right)^{\beta}\left(T^{\rho}-t_{2}^{\rho}\right)}{\rho^{\beta+1} \Gamma(\beta+2)}-\frac{\mu\left(\xi^{\rho}-a^{\rho}\right)^{\beta+\gamma}\left[(\beta+1)\left(\xi^{\rho}-t_{2}^{\rho}\right)-\gamma\left(t^{\rho}-a^{\rho}\right)\right]}{\rho^{\beta+\gamma+1} \Gamma(\beta+\gamma+2)(\beta+1)}\right]\right. \\
& \left.-\frac{\left(t_{1}^{\rho}-a^{\rho}\right)^{\beta}}{\Omega\left(\eta^{\rho}-a^{\rho}\right)^{\beta}}\left[\frac{\left(T^{\rho}-a^{\rho}\right)^{\beta}\left(T^{\rho}-t_{1}^{\rho}\right)}{\rho^{\beta+1} \Gamma(\beta+2)}-\frac{\mu\left(\xi^{\rho}-a^{\rho}\right)^{\beta+\gamma}\left[(\beta+1)\left(\xi^{\rho}-t_{1}^{\rho}\right)-\gamma\left(t_{1}^{\rho}-a^{\rho}\right)\right]}{\rho^{\beta+\gamma+1} \Gamma(\beta+\gamma+2)(\beta+1)}\right]\right] \times \\
& \times^{\rho} I_{a+}^{\alpha+\beta} v(\eta) \\
& +\mid \frac{-\lambda \rho^{1-\beta}}{\Gamma(\beta)}\left[\int_{0}^{t_{1}} s^{\rho-1}\left[\left(t_{2}^{\rho}-s^{\rho}\right)^{\beta-1}-\left(t_{1}^{\rho}-s^{\rho}\right)^{\beta-1}\right] x(s) d s+\int_{t_{1}}^{t_{2}} s^{\rho-1}\left(t_{2}^{\rho}-s^{\rho}\right)^{\beta-1} x(s) d s\right] \\
& +\left[\frac{\left(t_{2}^{\rho}-a^{\rho}\right)^{\beta}\left(\eta^{\rho}-t_{2}^{\rho}\right)}{\rho^{\beta+1} \Gamma(\beta+2) \Omega}-\frac{\left(t_{1}^{\rho}-a^{\rho}\right)^{\beta}\left(\eta^{\rho}-t_{1}^{\rho}\right)}{\rho^{\beta+1} \Gamma(\beta+2) \Omega}\right]\left\{-\lambda^{\rho} I_{a+}^{\beta} x(T)+\mu \lambda^{\rho} I_{a+}^{\beta+\gamma} x(\xi)\right\} \\
& +\left[\frac{\lambda\left(t_{2}^{\rho}-a^{\rho}\right)^{\beta}}{\Omega\left(\eta^{\rho}-a^{\rho}\right)^{\beta}}\left[\frac{\left(T^{\rho}-a^{\rho}\right)^{\beta}\left(T^{\rho}-t_{2}^{\rho}\right)}{\rho^{\beta+1} \Gamma(\beta+2)}-\frac{\mu\left(\xi^{\rho}-a^{\rho}\right)^{\beta+\gamma}\left[(\beta+1)\left(\xi^{\rho}-t_{2}^{\rho}\right)-\gamma\left(t^{\rho}-a^{\rho}\right)\right]}{\rho^{\beta+\gamma+1} \Gamma(\beta+\gamma+2)(\beta+1)}\right]\right. \\
& \left.-\frac{\lambda\left(t_{1}^{\rho}-a^{\rho}\right)^{\beta}}{\Omega\left(\eta^{\rho}-a^{\rho}\right)^{\beta}}\left[\frac{\left(T^{\rho}-a^{\rho}\right)^{\beta}\left(T^{\rho}-t_{1}^{\rho}\right)}{\rho^{\beta+1} \Gamma(\beta+2)}-\frac{\mu\left(\xi^{\rho}-a^{\rho}\right)^{\beta+\gamma}\left[(\beta+1)\left(\xi^{\rho}-t_{1}^{\rho}\right)-\gamma\left(t_{1}^{\rho}-a^{\rho}\right)\right]}{\rho^{\beta+\gamma+1} \Gamma(\beta+\gamma+2)(\beta+1)}\right]\right] \times \\
& x^{\rho} I_{a+}^{\beta} x(\eta) \\
& \leq \frac{\|P\| Q(r)}{\rho^{\alpha+\beta} \Gamma(\alpha+\beta+1)}\left\{\left|t_{2}^{\rho(\alpha+\beta)}-t_{1}^{\rho(\alpha+\beta)}\right|+2\left(t_{2}^{\rho}-t_{1}^{\rho}\right)^{\alpha+\beta}\right\} \\
& +\left|\frac{\left(t_{2}^{\rho}-a^{\rho}\right)^{\beta}\left(\eta^{\rho}-t_{2}^{\rho}\right)}{\rho^{\beta+1} \Gamma(\beta+2) \Omega}-\frac{\left(t_{1}^{\rho}-a^{\rho}\right)^{\beta}\left(\eta^{\rho}-t_{1}^{\rho}\right)}{\rho^{\beta+1} \Gamma(\beta+2) \Omega}\right| \\
& \times\|P\| Q(r)\left(\frac{\left(T^{\rho}-a^{\rho}\right)^{\alpha+\beta}}{\rho^{\alpha+\beta} \Gamma(\alpha+\beta+1)}+|\mu| \frac{\left(\xi^{\rho}-a^{\rho}\right)^{\alpha+\beta+\gamma}}{\rho^{\alpha+\beta+\gamma} \Gamma(\alpha \beta+\gamma+1)}\right) \\
& +\mid \frac{\left(t_{2}^{\rho}-a^{\rho}\right)^{\beta}}{\Omega\left(\eta^{\rho}-a^{\rho}\right)^{\beta}}\left[\frac{\left(T^{\rho}-a^{\rho}\right)^{\beta}\left(T^{\rho}-t_{2}^{\rho}\right)}{\rho^{\beta+1} \Gamma(\beta+2)}-\frac{\mu\left(\xi^{\rho}-a^{\rho}\right)^{\beta+\gamma}\left[(\beta+1)\left(\xi^{\rho}-t_{2}^{\rho}\right)-\gamma\left(t_{2}^{\rho}-a^{\rho}\right)\right]}{\rho^{\beta+\gamma+1} \Gamma(\beta+\gamma+2)(\beta+1)}\right] \\
& -\frac{\left(t_{1}^{\rho}-a^{\rho}\right)^{\beta}}{\Omega\left(\eta^{\rho}-a^{\rho}\right)^{\beta}}\left[\frac{\left(T^{\rho}-a^{\rho}\right)^{\beta}\left(T^{\rho}-t_{1}^{\rho}\right)}{\rho^{\beta+1} \Gamma(\beta+2)}-\frac{\mu\left(\xi^{\rho}-a^{\rho}\right)^{\beta+\gamma}\left[(\beta+1)\left(\xi^{\rho}-t_{1}^{\rho}\right)-\gamma\left(t_{1}^{\rho}-a^{\rho}\right)\right]}{\rho^{\beta+\gamma+1} \Gamma(\beta+\gamma+2)(\beta+1)}\right] \mid \times \\
& \times\|P\| Q(r) \frac{\left(\eta^{\rho}-a^{\rho}\right)^{\alpha+\beta}}{\rho^{\alpha+\beta} \Gamma(\alpha+\beta+1)}+\frac{r}{\rho^{\beta} \Gamma(\beta+1)}\left\{\left|t_{2}^{\rho \beta}-t_{1}^{\rho \beta}\right|+2\left(t_{2}^{\rho}-t_{1}^{\rho}\right)^{\beta}\right\} \\
& +\left|\left[\frac{\left(t_{2}^{\rho}-a^{\rho}\right)^{\beta}\left(\eta^{\rho}-t_{2}^{\rho}\right)}{\rho^{\beta+1} \Gamma(\beta+2) \Omega}-\frac{\left(t_{1}^{\rho}-a^{\rho}\right)^{\beta}\left(\eta^{\rho}-t_{1}^{\rho}\right)}{\rho^{\beta+1} \Gamma(\beta+2) \Omega}\right]\right||\lambda| r\left(\frac{\left(T^{\rho}-a^{\rho}\right)^{\beta}}{\rho^{\beta} \Gamma(\beta+1)}+|\mu| \frac{\left(\xi^{\rho}-a^{\rho}\right)^{\beta+\gamma}}{\rho^{\beta+\gamma} \Gamma(\beta+\gamma+1)}\right) \\
& +\mid \frac{\lambda\left(t_{2}^{\rho}-a^{\rho}\right)^{\beta}}{\Omega\left(\eta^{\rho}-a^{\rho}\right)^{\beta}}\left[\frac{\left(T^{\rho}-a^{\rho}\right)^{\beta}\left(T^{\rho}-t_{2}^{\rho}\right)}{\rho^{\beta+1} \Gamma(\beta+2)}-\frac{\mu\left(\xi^{\rho}-a^{\rho}\right)^{\beta+\gamma}\left[(\beta+1)\left(\xi^{\rho}-t_{2}^{\rho}\right)-\gamma\left(t^{\rho}-a^{\rho}\right)\right]}{\rho^{\beta+\gamma+1} \Gamma(\beta+\gamma+2)(\beta+1)}\right] \\
& -\frac{\lambda\left(t_{1}^{\rho}-a^{\rho}\right)^{\beta}}{\Omega\left(\eta^{\rho}-a^{\rho}\right)^{\beta}}\left[\frac{\left(T^{\rho}-a^{\rho}\right)^{\beta}\left(T^{\rho}-t_{1}^{\rho}\right)}{\rho^{\beta+1} \Gamma(\beta+2)}-\frac{\mu\left(\xi^{\rho}-a^{\rho}\right)^{\beta+\gamma}\left[(\beta+1)\left(\xi^{\rho}-t_{1}^{\rho}\right)-\gamma\left(t_{1}^{\rho}-a^{\rho}\right)\right]}{\rho^{\beta+\gamma+1} \Gamma(\beta+\gamma+2)(\beta+1)}\right] \mid \times \\
& \times \frac{\left(\eta^{\rho}-a^{\rho}\right)^{\beta}}{\rho^{\beta} \Gamma(\beta+1)} \rightarrow 0 \text { when } t_{1} \rightarrow t_{2} \text {, independently of } x \in B_{r} \text {. }
\end{aligned}
$$

Combining the outcome of Steps 1-3 with Arzelá-Ascoli theorem leads to the conclusion that $N: C(J, \mathbb{R}) \rightarrow \mathcal{P}(C(J, \mathbb{R}))$ is completely continuous.

Next, we show that $N$ has a closed graph. Then it will follow by Proposition 1.2 in [19] that the operator $N$ is u.s.c. 
Step 4. $N$ has a closed graph.

Suppose that there exists $x_{n} \rightarrow x_{*}, h_{n} \in N\left(x_{n}\right)$ and $h_{n} \rightarrow h_{*}$. Then we have to establish that $h_{*} \in N\left(x_{*}\right)$. Since $h_{n} \in N\left(x_{n}\right)$, there exists $v_{n} \in S_{F, x_{n}}$. In consequence, for each $t \in J$, we get

$$
\begin{aligned}
h_{n}(t)= & \rho I_{a+}^{\alpha+\beta} v_{n}(t)-\lambda^{\rho} I_{a+}^{\beta} x_{n}(t)+\frac{\left(t^{\rho}-a^{\rho}\right)^{\beta}\left(\eta^{\rho}-t^{\rho}\right)}{\rho^{\beta+1} \Gamma(\beta+2) \Omega}\left\{{ }^{\rho} I_{a+}^{\alpha+\beta} v_{n}(T)-\lambda^{\rho} I_{a+}^{\beta} x_{n}(T)\right. \\
& \left.-\mu^{\rho} I_{a+}^{\alpha+\beta+\gamma} v_{n}(\xi)+\mu \lambda^{\rho} I_{a+}^{\beta+\gamma} x_{n}(\xi)\right\}-\frac{\left(t^{\rho}-a^{\rho}\right)^{\beta}}{\Omega\left(\eta^{\rho}-a^{\rho}\right)^{\beta}}\left(\frac{\left(T^{\rho}-a^{\rho}\right)^{\beta}\left(T^{\rho}-t^{\rho}\right)}{\rho^{\beta+1} \Gamma(\beta+2)}\right. \\
& \left.-\frac{\mu\left(\xi^{\rho}-a^{\rho}\right)^{\beta+\gamma}\left[(\beta+1)\left(\xi^{\rho}-t^{\rho}\right)-\gamma\left(t^{\rho}-a^{\rho}\right)\right]}{\rho^{\beta+\gamma+1} \Gamma(\beta+\gamma+2)(\beta+1)}\right)\left\{{ }^{\rho} I_{a+}^{\alpha+\beta} v_{n}(\eta)-\lambda^{\rho} I_{a+}^{\beta} x_{n}(\eta)\right\} .
\end{aligned}
$$

Next we show that there exists $v_{*} \in S_{F, x_{*}}$ such that, for each $t \in J$,

$$
\begin{aligned}
h_{*}(t)= & { }^{\rho} I_{a+}^{\alpha+\beta} v_{*}(t)-\lambda^{\rho} I_{a+}^{\beta} x_{*}(t)+\frac{\left(t^{\rho}-a^{\rho}\right)^{\beta}\left(\eta^{\rho}-t^{\rho}\right)}{\rho^{\beta+1} \Gamma(\beta+2) \Omega}\left\{{ }^{\rho} I_{a+}^{\alpha+\beta} v_{*}(T)-\lambda^{\rho} I_{a+}^{\beta} x_{*}(T)\right. \\
& \left.-\mu^{\rho} I_{a+}^{\alpha+\beta+\gamma} v_{*}(\xi)+\mu \lambda^{\rho} I_{a+}^{\beta+\gamma} x_{*}(\xi)\right\}-\frac{\left(t^{\rho}-a^{\rho}\right)^{\beta}}{\Omega\left(\eta^{\rho}-a^{\rho}\right)^{\beta}}\left(\frac{\left(T^{\rho}-a^{\rho}\right)^{\beta}\left(T^{\rho}-t^{\rho}\right)}{\rho^{\beta+1} \Gamma(\beta+2)}\right. \\
& \left.-\frac{\mu\left(\xi^{\rho}-a^{\rho}\right)^{\beta+\gamma}\left[(\beta+1)\left(\xi^{\rho}-t^{\rho}\right)-\gamma\left(t^{\rho}-a^{\rho}\right)\right]}{\rho^{\beta+\gamma+1} \Gamma(\beta+\gamma+2)(\beta+1)}\right)\left\{{ }^{\rho} I_{a+}^{\alpha+\beta} v_{*}(\eta)-\lambda^{\rho} I_{a+}^{\beta} x_{*}(\eta)\right\} .
\end{aligned}
$$

Consider the continuous linear operator $\Theta: L^{1}(J, \mathbb{R}) \rightarrow C(J, \mathbb{R})$ given by

$$
\begin{aligned}
v \rightarrow \Theta(v)(t)= & { }^{\rho} I_{a+}^{\alpha+\beta} v(t)-\lambda^{\rho} I_{a+}^{\beta} x(t)+\frac{\left(t^{\rho}-a^{\rho}\right)^{\beta}\left(\eta^{\rho}-t^{\rho}\right)}{\rho^{\beta+1} \Gamma(\beta+2) \Omega}\left\{{ }^{\rho} I_{a+}^{\alpha+\beta} v(T)-\lambda^{\rho} I_{a+}^{\beta} x(T)\right. \\
& \left.-\mu^{\rho} I_{a+}^{\alpha+\beta+\gamma} v(\xi)+\mu \lambda^{\rho} I_{a+}^{\beta+\gamma} x(\xi)\right\}-\frac{\left(t^{\rho}-a^{\rho}\right)^{\beta}}{\Omega\left(\eta^{\rho}-a^{\rho}\right)^{\beta}}\left(\frac{\left(T^{\rho}-a^{\rho}\right)^{\beta}\left(T^{\rho}-t^{\rho}\right)}{\rho^{\beta+1} \Gamma(\beta+2)}\right. \\
& \left.-\frac{\mu\left(\xi^{\rho}-a^{\rho}\right)^{\beta+\gamma}\left[(\beta+1)\left(\xi^{\rho}-t^{\rho}\right)-\gamma\left(t^{\rho}-a^{\rho}\right)\right]}{\rho^{\beta+\gamma+1} \Gamma(\beta+\gamma+2)(\beta+1)}\right)\left\{{ }^{\rho} I_{a+}^{\alpha+\beta} v(\eta)-\lambda^{\rho} I_{a+}^{\beta} x(\eta)\right\} .
\end{aligned}
$$

Notice that $\left\|h_{n}(t)-h_{*}(t)\right\| \rightarrow 0$ as $n \rightarrow \infty$. So we deduce by a closed graph result obtained in [20] that $\Theta \circ S_{F, x}$ is a closed graph operator. Furthermore, $h_{n} \in \Theta\left(S_{F, x_{n}}\right)$. Since $x_{n} \rightarrow x_{*}$, therefore we have

$$
\begin{aligned}
h_{*}(t)= & \rho I_{a+}^{\alpha+\beta} v_{*}(t)-\lambda^{\rho} I_{a+}^{\beta} x_{*}(t)+\frac{\left(t^{\rho}-a^{\rho}\right)^{\beta}\left(\eta^{\rho}-t^{\rho}\right)}{\rho^{\beta+1} \Gamma(\beta+2) \Omega}\left\{{ }^{\rho} I_{a+}^{\alpha+\beta} v_{*}(T)-\lambda^{\rho} I_{a+}^{\beta} x_{*}(T)\right. \\
& \left.-\mu^{\rho} I_{a+}^{\alpha+\beta+\gamma} v_{*}(\xi)+\mu \lambda^{\rho} I_{a+}^{\beta+\gamma} x_{*}(\xi)\right\}-\frac{\left(t^{\rho}-a^{\rho}\right)^{\beta}}{\Omega\left(\eta^{\rho}-a^{\rho}\right)^{\beta}}\left(\frac{\left(T^{\rho}-a^{\rho}\right)^{\beta}\left(T^{\rho}-t^{\rho}\right)}{\rho^{\beta+1} \Gamma(\beta+2)}\right. \\
& \left.-\frac{\mu\left(\xi^{\rho}-a^{\rho}\right)^{\beta+\gamma}\left[(\beta+1)\left(\xi^{\rho}-t^{\rho}\right)-\gamma\left(t^{\rho}-a^{\rho}\right)\right]}{\rho^{\beta+\gamma+1} \Gamma(\beta+\gamma+2)(\beta+1)}\right)\left\{{ }^{\rho} I_{a+}^{\alpha+\beta} v_{*}(\eta)-\lambda^{\rho} I_{a+}^{\beta} x_{*}(\eta)\right\},
\end{aligned}
$$

for some $v_{*} \in S_{F, x_{*}}$

Step 5. There exists an open set $\mathcal{V} \subseteq C(J, \mathbb{R})$ with $x \notin \theta N(x)$ for any $\theta \in(0,1)$ and all $x \in \partial \mathcal{V}$.

Take $\theta \in(0,1), x \in \theta N(x)$ and $t \in J$. Then we show that there exists $v \in L^{1}(J, \mathbb{R})$ with $v \in S_{F, x}$ such that

$$
\begin{aligned}
x(t)= & \theta^{\rho} I_{a+}^{\alpha+\beta} v(t)-\theta \lambda^{\rho} I_{a+}^{\beta} x(t)+\theta \frac{\left(t^{\rho}-a^{\rho}\right)^{\beta}\left(\eta^{\rho}-t^{\rho}\right)}{\rho^{\beta+1} \Gamma(\beta+2) \Omega}\left\{{ }^{\rho} I_{a+}^{\alpha+\beta} v_{*}(T)-\lambda^{\rho} I_{a+}^{\beta} x(T)\right. \\
& \left.-\mu^{\rho} I_{a+}^{\alpha+\beta+\gamma} v(\xi)+\mu \lambda^{\rho} I_{a+}^{\beta+\gamma} x(\xi)\right\}-\theta \frac{\left(t^{\rho}-a^{\rho}\right)^{\beta}}{\Omega\left(\eta^{\rho}-a^{\rho}\right)^{\beta}}\left(\frac{\left(T^{\rho}-a^{\rho}\right)^{\beta}\left(T^{\rho}-t^{\rho}\right)}{\rho^{\beta+1} \Gamma(\beta+2)}\right. \\
& \left.-\frac{\mu\left(\xi^{\rho}-a^{\rho}\right)^{\beta+\gamma}\left[(\beta+1)\left(\xi^{\rho}-t^{\rho}\right)-\gamma\left(t^{\rho}-a^{\rho}\right)\right]}{\rho^{\beta+\gamma+1} \Gamma(\beta+\gamma+2)(\beta+1)}\right)\left\{{ }^{\rho} I_{a+}^{\alpha+\beta} v(\eta)-\lambda^{\rho} I_{a+}^{\beta} x(\eta)\right\} .
\end{aligned}
$$


Using the computations done in Step 2, for each $t \in J$, we get

$$
|x(t)| \leq \Lambda_{1}\|P\| Q(\|x\|)+\Lambda_{2}\|x\|,
$$

which yields

$$
\frac{\left(1-\Lambda_{2}\right)\|x\|}{\Lambda_{1}\|P\| Q(\|x\|)} \leq 1
$$

By $\left(A_{3}\right)$, there exists $M$ such that $\|x\| \neq M$. Define a set

$$
\mathcal{V}=\{x \in C(J, \mathbb{R}):\|x\|<M\} .
$$

Observe that the operator $N: \overline{\mathcal{V}} \rightarrow \mathcal{P}(C(J, \mathbb{R}))$ is a compact multivalued map, u.s.c. with convex closed values. With the given choice of $\mathcal{V}$, it is not possible to find $x \in \partial \mathcal{V}$ satisfying $x \in \theta N(x)$ for some $\theta \in(0,1)$. Consequently, by the nonlinear alternative of Leray-Schauder type [18], the operator $N$ has a fixed point $x \in \overline{\mathcal{V}}$, which corresponds to a solution of the problem (1). This finishes the proof.

\subsection{The Lipschitz Case}

Let $(\mathcal{X}, d)$ denote a metric space induced from the normed space $(\mathcal{X} ;\|\cdot\|)$. Let $H_{d}: \mathcal{P}(\mathcal{X}) \times$ $\mathcal{P}(\mathcal{X}) \rightarrow \mathbb{R} \cup\{\infty\}$ be defined by $H_{d}\left(A_{1}, A_{2}\right)=\max \left\{\sup _{a_{1} \in A_{1}} d\left(a_{1}, A_{2}\right), \sup _{a_{2} \in A_{2}} d\left(A_{1}, a_{2}\right)\right\}$, where $d\left(A_{1}, a_{2}\right)=\inf _{a_{1} \in A_{1}} d\left(a_{1} ; a_{2}\right)$ and $d\left(a_{1}, A_{2}\right)=\inf _{a_{2} \in A_{2}} d\left(a_{1} ; a_{2}\right)$. Then $\left(\mathcal{P}_{b, c l}(\mathcal{X}), H_{d}\right)$ is a metric space (see [21]), where $\mathcal{P}_{b, c l}(\mathcal{X})=\{\mathcal{Y} \in \mathcal{P}(\mathcal{X}): \mathcal{Y}$ is bounded and closed $\}$,

The following result deals with the non-convex valued case of the problem (1) and is based on Covitz and Nadler's fixed point theorem [22]: "If $N: \mathcal{X} \rightarrow \mathcal{P}_{c l}(\mathcal{X})$ is a contraction, then Fix $N \neq \varnothing$, where $\mathcal{P}_{c l}(\mathcal{X})=\{\mathcal{Y} \in \mathcal{P}(\mathcal{X}): \mathcal{Y} \text { is closed }\}^{\prime \prime}$.

Theorem 2. Assume that

$\left(A_{4}\right) F: J \times \mathbb{R} \rightarrow \mathcal{P}_{c p}(\mathbb{R})$ is such that $F(\cdot, x): J \rightarrow \mathcal{P}_{c p}(\mathbb{R})$ is measurable for each $x \in \mathbb{R}$, where $\mathcal{P}_{c p}(\mathbb{R})=\{\mathcal{Y} \in \mathcal{P}(\mathbb{R}): \mathcal{Y}$ is compact $\}$

$\left(A_{5}\right) H_{d}(F(t, x), F(t, \widehat{x})) \leq \omega(t)|x-\widehat{x}|$ for almost all $t \in J$ and $x, \widehat{x} \in \mathbb{R}$ with $\omega \in C\left(J, \mathbb{R}^{+}\right)$and $d(0, F(t, 0)) \leq \omega(t)$ for almost all $t \in J$.

Then the problem (1) has at least one solution on $J$ if

$$
\|\omega\| \Lambda_{1}+\Lambda_{2}<1
$$

where $\Lambda_{1}$ and $\Lambda_{2}$ are respectively given by (14) and (15).

Proof. Let us verify that the operator $N: C(J, \mathbb{R}) \rightarrow \mathcal{P}(C(J, \mathbb{R}))$, defined in the proof of the last theorem, satisfies the hypothesis of Covitz and Nadler fixed point theorem [22]. We establish it in two steps.

Step I. $N(x)$ is nonempty and closed for every $v \in S_{F, x}$.

Since the set-valued map $F(\cdot, x(\cdot))$ is measurable, it admits a measurable selection $v: J \rightarrow \mathbb{R}$ by the measurable selection theorem ([23], Theorem III.6). By $\left(A_{5}\right)$, we have

$$
|v(t)| \leq \omega(t)(1+|x(t)|),
$$

that is, $v \in L^{1}(J, \mathbb{R})$. So $F$ is integrably bounded. Therefore, $S_{F, x} \neq \varnothing$. 
Now we establish that $N(x)$ is closed for each $x \in C(J, \mathbb{R})$. Let $\left\{u_{n}\right\}_{n \geq 0} \in N(x)$ be such that $u_{n} \rightarrow u$ as $n \rightarrow \infty$ in $C(J, \mathbb{R})$. Then $u \in C(J, \mathbb{R})$ and we can find $v_{n} \in S_{F, x_{n}}$ such that, for each $t \in J$,

$$
\begin{aligned}
u_{n}(t)= & { }^{\rho} I_{a+}^{\alpha+\beta} v_{n}(t)-\lambda^{\rho} I_{a+}^{\beta} x_{n}(t)+\frac{\left(t^{\rho}-a^{\rho}\right)^{\beta}\left(\eta^{\rho}-t^{\rho}\right)}{\rho^{\beta+1} \Gamma(\beta+2) \Omega}\left\{{ }^{\rho} I_{a+}^{\alpha+\beta} v(T)-\lambda^{\rho} I_{a+}^{\beta} x_{n}(T)\right. \\
& \left.-\mu^{\rho} I_{a+}^{\alpha+\beta+\gamma} v_{n}(\xi)+\mu \lambda^{\rho} I_{a+}^{\beta+\gamma} x_{n}(\xi)\right\}-\frac{\left(t^{\rho}-a^{\rho}\right)^{\beta}}{\Omega\left(\eta^{\rho}-a^{\rho}\right)^{\beta}}\left(\frac{\left(T^{\rho}-a^{\rho}\right)^{\beta}\left(T^{\rho}-t^{\rho}\right)}{\rho^{\beta+1} \Gamma(\beta+2)}\right. \\
& \left.-\frac{\mu\left(\xi^{\rho}-a^{\rho}\right)^{\beta+\gamma}\left[(\beta+1)\left(\xi^{\rho}-t^{\rho}\right)-\gamma\left(t^{\rho}-a^{\rho}\right)\right]}{\rho^{\beta+\gamma+1} \Gamma(\beta+\gamma+2)(\beta+1)}\right)\left\{{ }^{\rho} I_{a+}^{\alpha+\beta} v_{n}(\eta)-\lambda^{\rho} I_{a+}^{\beta} x_{n}(\eta)\right\} .
\end{aligned}
$$

As $F$ has compact values, we can pass onto a subsequence (if necessary) to obtain that $v_{n}$ converges to $v$ in $L^{1}(J, \mathbb{R})$. So $v \in S_{F, x}$. Then, for each $t \in J$, we get

$$
\begin{aligned}
u_{n}(t) \rightarrow v(t)= & { }^{\rho} I_{a+}^{\alpha+\beta} v(t)-\lambda^{\rho} I_{a+}^{\beta} x(t)+\frac{\left(t^{\rho}-a^{\rho}\right)^{\beta}\left(\eta^{\rho}-t^{\rho}\right)}{\rho^{\beta+1} \Gamma(\beta+2) \Omega}\left\{{ }^{\rho} I_{a+}^{\alpha+\beta} v(T)-\lambda^{\rho} I_{a+}^{\beta} x(T)\right. \\
& \left.-\mu^{\rho} I_{a+}^{\alpha+\beta+\gamma} v(\xi)+\mu \lambda^{\rho} I_{a+}^{\beta+\gamma} x(\xi)\right\}-\frac{\left(t^{\rho}-a^{\rho}\right)^{\beta}}{\Omega\left(\eta^{\rho}-a^{\rho}\right)^{\beta}}\left(\frac{\left(T^{\rho}-a^{\rho}\right)^{\beta}\left(T^{\rho}-t^{\rho}\right)}{\rho^{\beta+1} \Gamma(\beta+2)}\right. \\
& \left.-\frac{\mu\left(\xi^{\rho}-a^{\rho}\right)^{\beta+\gamma}\left[(\beta+1)\left(\xi^{\rho}-t^{\rho}\right)-\gamma\left(t^{\rho}-a^{\rho}\right)\right]}{\rho^{\beta+\gamma+1} \Gamma(\beta+\gamma+2)(\beta+1)}\right)\left\{{ }^{\rho} I_{a+}^{\alpha+\beta} v(\eta)-\lambda^{\rho} I_{a+}^{\beta} x(\eta)\right\},
\end{aligned}
$$

which implies that $u \in N(x)$.

Step II. We establish that there exists $0<\hat{\theta}<1\left(\hat{\theta}=\Lambda_{1}\|\mathcal{}\|+\Lambda_{2}\right)$ satisfying

$$
H_{d}(N(x), N(\widehat{x})) \leq \hat{\theta}\|x-\widehat{x}\| \text { for each } x, \widehat{x} \in C(J, \mathbb{R}) .
$$

Let us take $x, \widehat{x} \in C(J, \mathbb{R})$ and $h_{1} \in N(x)$. Then there exists $v_{1}(t) \in F(t, x(t))$ such that, for each $t \in J$,

$$
\begin{aligned}
h_{1}(t)= & I_{a+}^{\alpha+\beta} v_{1}(t)-\lambda^{\rho} I_{a+}^{\beta} x(t)+\frac{\left(t^{\rho}-a^{\rho}\right)^{\beta}\left(\eta^{\rho}-t^{\rho}\right)}{\rho^{\beta+1} \Gamma(\beta+2) \Omega}\left\{{ }^{\rho} I_{a+}^{\alpha+\beta} v_{1}(T)-\lambda^{\rho} I_{a+}^{\beta} x(T)\right. \\
& \left.-\mu^{\rho} I_{a+}^{\alpha+\beta+\gamma} v_{1}(\xi)+\mu \lambda^{\rho} I_{a+}^{\beta+\gamma} x(\xi)\right\}-\frac{\left(t^{\rho}-a^{\rho}\right)^{\beta}}{\Omega\left(\eta^{\rho}-a^{\rho}\right)^{\beta}}\left(\frac{\left(T^{\rho}-a^{\rho}\right)^{\beta}\left(T^{\rho}-t^{\rho}\right)}{\rho^{\beta+1} \Gamma(\beta+2)}\right. \\
& \left.-\frac{\mu\left(\xi^{\rho}-a^{\rho}\right)^{\beta+\gamma}\left[(\beta+1)\left(\xi^{\rho}-t^{\rho}\right)-\gamma\left(t^{\rho}-a^{\rho}\right)\right]}{\rho^{\beta+\gamma+1} \Gamma(\beta+\gamma+2)(\beta+1)}\right)\left\{{ }^{\rho} I_{a+}^{\alpha+\beta} v_{1}(\eta)-\lambda^{\rho} I_{a+}^{\beta} x(\eta)\right\} .
\end{aligned}
$$

By $\left(A_{5}\right)$, we have that $H_{d}(F(t, x), F(t, \widehat{x})) \leq \mathfrak{W}(t)|x(t)-\widehat{x}(t)|$. So, there exists $w(t) \in F(t, \widehat{x}(t))$ satisfying $\left|v_{1}(t)-w\right| \leq \mathfrak{\omega}(t)|x(t)-\widehat{x}(t)|, \quad t \in J$.

Define $\mathcal{W}: J \rightarrow \mathcal{P}(\mathbb{R})$ by

$$
\mathcal{W}(t)=\left\{w \in \mathbb{R}:\left|v_{1}(t)-w\right| \leq \mathfrak{\omega}(t)|x(t)-\widehat{x}(t)|\right\} .
$$

As the multivalued operator $\mathcal{W}(t) \cap F(t, \widehat{x}(t))$ is measurable by Proposition III.4 [23], we can find a function $v_{2}(t)$ which is a measurable selection for $\mathcal{W}$. So $v_{2}(t) \in F(t, \widehat{x}(t))$ and for each $t \in J$, we have $\left|v_{1}(t)-v_{2}(t)\right| \leq \omega(t)|x(t)-\widehat{x}(t)|$. For each $t \in J$, we define

$$
\begin{aligned}
h_{2}(t)= & { } I_{a+}^{\alpha+\beta} v_{2}(t)-\lambda^{\rho} I_{a+}^{\beta} \widehat{x}(t)+\frac{\left(t^{\rho}-a^{\rho}\right)^{\beta}\left(\eta^{\rho}-t^{\rho}\right)}{\rho^{\beta+1} \Gamma(\beta+2) \Omega}\left\{{ }^{\rho} I_{a+}^{\alpha+\beta} v_{2}(T)-\lambda^{\rho} I_{a+}^{\beta} \widehat{x}(T)\right. \\
& \left.-\mu^{\rho} I_{a+}^{\alpha+\beta+\gamma} v_{2}(\xi)+\mu \lambda^{\rho} I_{a+}^{\beta+\gamma} \widehat{x}(\xi)\right\}-\frac{\left(t^{\rho}-a^{\rho}\right)^{\beta}}{\Omega\left(\eta^{\rho}-a^{\rho}\right)^{\beta}}\left(\frac{\left(T^{\rho}-a^{\rho}\right)^{\beta}\left(T^{\rho}-t^{\rho}\right)}{\rho^{\beta+1} \Gamma(\beta+2)}\right. \\
& \left.-\frac{\mu\left(\xi^{\rho}-a^{\rho}\right)^{\beta+\gamma}\left[(\beta+1)\left(\xi^{\rho}-t^{\rho}\right)-\gamma\left(t^{\rho}-a^{\rho}\right)\right]}{\rho^{\beta+\gamma+1} \Gamma(\beta+\gamma+2)(\beta+1)}\right)\left\{{ }^{\rho} I_{a+}^{\alpha+\beta} v_{2}(\eta)-\lambda^{\rho} I_{a+}^{\beta} \widehat{x}(\eta)\right\} .
\end{aligned}
$$


As a result, we get

$$
\begin{aligned}
& \left|h_{1}(t)-h_{2}(t)\right| \\
= & \mid \rho I_{a+}^{\alpha+\beta}\left[v_{2}(s)-v_{1}(s)\right](t)-\lambda^{\rho} I_{a+}^{\beta}[x(t)-\widehat{x}(t)] \\
& +\frac{\left(t^{\rho}-a^{\rho}\right)^{\beta}\left(\eta^{\rho}-t^{\rho}\right)}{\rho^{\beta+1} \Gamma(\beta+2) \Omega}\left\{{ }^{\rho} I_{a+}^{\alpha+\beta}\left[v_{2}(s)-v_{1}(s)\right](T)-\lambda^{\rho} I_{a+}^{\beta}[x(T)-\widehat{x}(T)]\right. \\
& \left.-\mu^{\rho} I_{a+}^{\alpha+\beta+\gamma}\left[v_{2}(s)-v_{1}(s)\right](\xi)+\mu \lambda^{\rho} I_{a+}^{\beta+\gamma}[x(\xi)-\widehat{x}(\xi)]\right\}-\frac{\left(t^{\rho}-a^{\rho}\right)^{\beta}}{\Omega\left(\eta^{\rho}-a^{\rho}\right)^{\beta}}\left(\frac{\left(T^{\rho}-a^{\rho}\right)^{\beta}\left(T^{\rho}-t^{\rho}\right)}{\rho^{\beta+1} \Gamma(\beta+2)}\right. \\
& \left.-\frac{\mu\left(\xi^{\rho}-a^{\rho}\right)^{\beta+\gamma}\left[(\beta+1)\left(\xi^{\rho}-t^{\rho}\right)-\gamma\left(t^{\rho}-a^{\rho}\right)\right]}{\rho^{\beta+\gamma+1} \Gamma(\beta+\gamma+2)(\beta+1)}\right)\left\{{ }^{\rho} I_{a+}^{\alpha+\beta}\left[v_{2}(s)-v_{1}(s)\right](\eta)-\lambda^{\rho} I_{a+}^{\beta}[x(\eta)-\widehat{x}(\eta)]\right\} \mid \\
\leq & \|\oplus\|\|x-\widehat{x}\|\left(\frac{\left(T^{\rho}-a^{\rho}\right)^{\alpha+\beta}}{\rho^{\alpha+\beta} \Gamma(\alpha+\beta+1)}\left[1+\frac{\zeta_{1}}{\rho^{\beta+1} \Gamma(\beta+2)|\Omega|}\right]\right. \\
& +\frac{|\mu|\left(\xi^{\rho}-a^{\rho}\right)^{\alpha+\beta+\gamma} \zeta_{1}}{\rho^{\alpha+2 \beta+\gamma+1} \Gamma(\alpha+\beta+\gamma+1) \Gamma(\beta+2)|\Omega|} \\
& \left.+\frac{\left(\eta^{\rho}-a^{\rho}\right)^{\alpha} \zeta_{2}}{\rho^{\alpha+\beta} \Gamma(\alpha+\beta+1)|\Omega|}\right)+\|x-\widehat{x}\|\left(\frac{|\lambda|\left(T^{\rho}-a^{\rho}\right)^{\beta}}{\rho^{\beta} \Gamma(\beta+1)}\left[1+\frac{\zeta_{1}}{\rho^{\beta+1} \Gamma(\beta+2)|\Omega|}\right]\right. \\
& \left.+\frac{|\mu \|| \lambda \mid\left(\xi^{\rho}-a^{\rho}\right)^{\beta+\gamma} \zeta_{1}}{\rho^{2 \beta+\gamma+1} \Gamma(\beta+\gamma+1) \Gamma(\beta+2)|\Omega|}+\frac{|\lambda| \zeta_{2}}{\rho^{\beta} \Gamma(\beta+1)|\Omega|}\right) \\
= & \left(\|\oplus\| \Lambda_{1}+\Lambda_{2}\right)\|x-\widehat{x}\| .
\end{aligned}
$$

Hence

$$
\left\|h_{1}-h_{2}\right\| \leq\left(\|\mathscr{}\| \Lambda_{1}+\Lambda_{2}\right)\|x-\widehat{x}\| .
$$

Analogously, we can interchange the roles of $x$ and $\widehat{x}$ to get

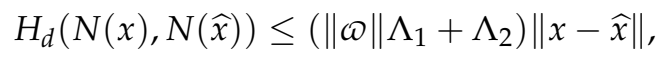

which implies that $N$ is a contraction by the condition (18). Hence, by the conclusion of Covitz and Nadler fixed point theorem [22], $N$ has a fixed point $x$, which corresponds to a solution of (1). This finishes the proof.

\section{Examples}

We illustrate our main results by presenting a numerical example.

Example 1. Consider the following problem

$$
\left\{\begin{array}{l}
{ }_{c}^{1 / 3} D^{5 / 4}\left({ }_{c}^{1 / 3} D^{1 / 4}+1 / 5\right) x(t) \in F(t, x(t)), \quad t \in J:=[1,2], \\
x(1)=0, \quad x(3 / 2)=0, \quad x(2)=2 / 7^{1 / 3} I^{3 / 4} x(7 / 4) .
\end{array}\right.
$$

Here $\rho=1 / 3, \alpha=5 / 4, \beta=1 / 4, \lambda=1 / 5, a=1, T=2 \eta=3 / 2, \mu=2 / 7, \gamma=3 / 4, \xi=7 / 4$. Using the given data, we find that $\zeta_{1} \approx 0.082260, \zeta_{2} \approx 0.232036,|\Omega| \approx 0.293634, \Lambda_{1} \approx 1.336009$ and $\Lambda_{2} \approx 0.673563$, where $\zeta_{1}, \zeta_{2}, \Lambda_{1}$ and $\Lambda_{2}$ are given by (16), (17), (14) and (15) respectively.

(i) Let us consider the function

$$
F(t, x(t))=\left[\frac{1}{\sqrt{t^{2}+63}}\left(\frac{|x(t)|}{3}\left(\frac{|x(t)|}{|x(t)|+1}+2\right)+1\right), \frac{e^{-t}}{9 t+8}\left(\sin x(t)+\frac{1}{80}\right)\right] .
$$

We note that $|F(t, x(t))| \leq P(t) Q(\|x\|)$, where $P(t)=\frac{1}{\sqrt{t^{2}+63}}, Q(\|x\|)=\|x\|+1$. So the assumption $\left(A_{2}\right)$ holds. Moreover, there exists $M>1.047447394$ satisfying $\left(A_{3}\right)$. Thus the hypothesis 
of Theorem 1 holds true and hence there exists at least one solution for the problem (19) with $F(t, x)$ given by $(20)$ on $[1,2]$.

(ii) To illustrate Theorem 2 we consider the function

$$
F(t, x)=\left[\frac{e^{-t}}{19+t}, \frac{1}{(t+4)^{2}}\left(x+\tan ^{-1}(x)+\frac{1}{15}\right)\right] .
$$

Clearly $H_{d}(F(t, x), F(t, \bar{x})) \leq \omega(t)|x-\bar{x}|$, where $\omega(t)=\frac{2}{(t+4)^{2}}$. Also $d(0, F(t, 0)) \leq \omega(t)$ for almost all $t \in[0,1]$ and $\Lambda_{1}\|\omega\|+\Lambda_{2} \approx 0.7804433180<1$. As the hypothesis of Theorem 2 is satisfied, therefore we conclude that the multivalued problem (19) with $F(t, x)$ given by (21) has at least one solution on $[1,2]$.

\section{Conclusions}

We have introduced a new class of multivalued (inclusions) boundary value problems on an arbitrary domain containing Caputo-type generalized fractional differential operators of different orders and a generalized integral operator. We have considered convex as well as non-convex valued cases for the multi-valued map involved in the given problem. Leray-Schauder nonlinear alternative for multivalued maps plays a central role in proving the existence of solutions for convex valued case of the given problem, while the existence result for the non-convex valued case is based on Covitz and Nadler fixed point theorem. The work presented in this paper is not only new in the given configuration, but will also lead to some new results as special cases. For example, fixing $\mu=0$ in the obtained results, we obtain the ones for nonlocal three-point boundary conditions: $x(a)=0, x(\eta)=0, x(T)=0,0<\eta<T$. For $\rho=1$, our results specialize to the ones for Liouville-Caputo type fractional differential inclusions complemented with nonlocal generalized integral boundary conditions on an arbitrary domain.

Author Contributions: Conceptualization, B.A. and M.A.; methodology, A.A. and S.K.N.; validation, A.A., B.A., M.A. and S.K.N.; formal analysis, A.A., B.A., M.A. and S.K.N.; writing-original draft preparation, M.A.; writing-review and editing, A.A., B.A. and S.K.N.; project administration, B.A.; funding acquisition, A.A.

Funding: This project was funded by the Deanship of Scientific Research (DSR), King Abdulaziz University, Jeddah, Saudi Arabia under grant no. (KEP-PhD-70-130-38).

Acknowledgments: This project was funded by the Deanship of Scientific Research (DSR), King Abdulaziz University, Jeddah, under grant no. (KEP-PhD-70-130-38). The authors, therefore, acknowledge with thanks DSR technical and financial support.

Conflicts of Interest: The authors declare no conflict of interest.

\section{References}

1. Zaslavsky, G.M. Hamiltonian Chaos and Fractional Dynamics; Oxford University Press: Oxford, UK, 2005.

2. Magin, R.L. Fractional Calculus in Bioengineering; Begell House Publishers: Danbury, CT, USA, 2006.

3. Kilbas, A.A.; Srivastava, H.M.; Trujillo, J.J. Theory and Applications of Fractional Differential Equations; North-Holland Mathematics Studies 204; Elsevier Science B.V.: Amsterdam, The Netherlands, 2006.

4. Diethelm, K. The Analysis of Fractional Differential Equations. An Application-Oriented Exposition Using Differential Operators of Caputo Type; Lecture Notes in Mathematics 2004; Springer: Berlin, Germany, 2010.

5. Javidi, M.; Ahmad, B. Dynamic analysis of time fractional order phytoplankton-toxic phytoplanktonzooplankton system. Ecol. Model. 2015, 318, 8-18. [CrossRef]

6. Fallahgoul, H.A.; Focardi, S.M.; Fabozzi, F.J. Fractional Calculus and Fractional Processes with Applications to Financial Economics. Theory and Application; Elsevier/Academic Press: London, UK, 2017.

7. Ahmad, B.; Alsaedi, A.; Ntouyas, S.K.; Tariboon, J. Hadamard-Type Fractional Differential Equations, Inclusions and Inequalities; Springer: Cham, Switzerland, 2017.

8. Ahmad, B.; Nieto, J.J.; Alsaedi, A.; El-Shahed, M. A study of nonlinear Langevin equation involving two fractional orders in different intervals. Nonlinear Anal. Real World Appl. 2012, 13, 599-606. [CrossRef] 
9. Wang, G.; Zhang, L.; Song, G. Boundary value problem of a nonlinear Langevin equation with two different fractional orders and impulses. Fixed Point Theory Appl. 2012, 2012, 200. [CrossRef]

10. Ahmad, B.; Ntouyas, S.K. New existence results for differential inclusions involving Langevin equation with two indices. J. Nonlinear Convex Anal. 2013, 14, 437-450.

11. Muensawat, T.; Ntouyas, S.K.; Tariboon, J. Systems of generalized Sturm-Liouville and Langevin fractional differential equations. Adv. Differ. Equ. 2017, 2017, 63. [CrossRef]

12. Fazli, H.; Nieto, J.J. Fractional Langevin equation with anti-periodic boundary conditions. Chaos Solitons Fractals 2018, 114, 332-337. [CrossRef]

13. Ahmad, B.; Alsaedi, A.; Salem, S. On a nonlocal integral boundary value problem of nonlinear Langevin equation with different fractional orders. Adv. Differ. Equ. 2019, 2019, 57. [CrossRef]

14. Ahmad, B.; Alghanmi, M.; Alsaedi, A.; Srivastava, H.; Ntouyas, K. The Langevin equation in terms of generalized Liouville-Caputo derivatives with nonlocal boundary conditions involving a generalized fractional integral. Mathematics 2019, 7, 533. [CrossRef]

15. Katugampola, U.N. New Approach to a generalized fractional integral. Appl. Math. Comput. 2015, 218, 860-865. [CrossRef]

16. Katugampola, U.N. A new approach to generalized fractional derivatives. Bull. Math. Anal. Appl. 2014, 6, 1-15.

17. Jarad, F.; Abdeljawad, T.; Baleanu, D. On the generalized fractional derivatives and their caputo modification. J. Nonlinear Sci. Appl. 2017, 10, 2607-2619. [CrossRef]

18. Granas, A.; Dugundji, J. Fixed Point Theory; Springer: New York, NY, USA, 2005.

19. Deimling, K. Multivalued Differential Equations; De Gruyter: Berlin, Germany, 1992.

20. Lasota, A.; Opial, Z. An application of the Kakutani-Ky Fan theorem in the theory of ordinary differential equations, Bull. Acad. Pol. Sci. Ser. Sci. Math. Astronom. Phys. 1965, 13, 781-786.

21. Kisielewicz, M. Differential Inclusions and Optimal Control; Kluwer: Dordrecht, The Netherlands, 1991.

22. Covitz, H.; Nadler, S.B., Jr. Multivalued contraction mappings in generalized metric spaces. ISRAEL J. Math. 1970, 8, 5-11. [CrossRef]

23. Castaing, C.; Valadier, M. Convex Analysis and Measurable Multifunctions; Lecture Notes in Mathematics 580; Springer: Berlin/Heidelberg, Germany; New York, NY, USA, 1977.

(C) 2019 by the authors. Licensee MDPI, Basel, Switzerland. This article is an open access article distributed under the terms and conditions of the Creative Commons Attribution (CC BY) license (http:/ / creativecommons.org/licenses/by/4.0/). 